\title{
Comments on "Robust and Reliable Estimation Via Unscented Recursive Nonlinear Dynamic Data Reconciliation"
}

Sridhar Ungarala

Cleveland State University

Follow this and additional works at: https://engagedscholarship.csuohio.edu/encbe_facpub

Part of the Process Control and Systems Commons

How does access to this work benefit you? Let us know!

\section{Publisher's Statement}

NOTICE: this is the author's version of a work that was accepted for publication in Journal of Process Control. Changes resulting from the publishing process, such as peer review, editing, corrections, structural formatting, and other quality control mechanisms may not be reflected in this document. Changes may have been made to this work since it was submitted for publication. A definitive version was subsequently published in Journal of Process Control, 19, 4, (April 2009) DOI 10.1016/j.jprocont.2008.08.001

\section{Original Citation}

Ungarala, S. (2009). Comments on "Robust and reliable estimation via unscented recursive nonlinear dynamic data reconciliation”. Journal of Process Control, 19(4), 717-718. doi:10.1016/

j.jprocont.2008.08.001

\section{Repository Citation}

Ungarala, Sridhar, "Comments on "Robust and Reliable Estimation Via Unscented Recursive Nonlinear Dynamic Data Reconciliation"'" (2009). Chemical \& Biomedical Engineering Faculty Publications. 48.

https://engagedscholarship.csuohio.edu/encbe_facpub/48

This Response or Comment is brought to you for free and open access by the Chemical \& Biomedical Engineering Department at EngagedScholarship@CSU. It has been accepted for inclusion in Chemical \& Biomedical Engineering Faculty Publications by an authorized administrator of EngagedScholarship@CSU. For more information, please contact library.es@csuohio.edu. 


\section{Comments on "Robust and reliable estimation via unscented recursive nonlinear dynamic data reconciliation"}

In their paper Vachhani, Narasimhan and Rengaswamy [Journal of Process Control 16 (2006) 1075-1086], proposed state estimation with constraints by solving least squares optimization with respect to arbitrary sigma points around the state. In this note it is shown that the unscented recursive nonlinear dynamic data reconciliation (URNDDR) approach fails to reduce to Kalman filter for unconstrained linear Gaussian systems.

In the description of the procedure of unscented Kalman filter (UKF), Vachhani et al. missed a key step. After the sigma points are propagated and the mean and covariance are calculated for the predicted state (Eqs. (13) and (14) on p. 1078), new sigma points must be calculated around the predicted mean using the predicted covariance matrix. If this step is not performed the covariance matrix of the system noise $Q$ does not affect the filter gain.

In the URNDDR formulation, the authors did not justify the basis for posing the least squares problem for solving the "optimal" sigma points (p. 1079). State estimation as optimization can be based on minimizing errors or maximizing the probability density with respect to the state. This least squares objective function has no basis to be applicable to arbitrarily chosen sigma points. Moreover, the equality and inequality constraints of the states are not in general applicable to the sigma points, especially if the constraints are multivariate relationships. Since the proposed URNDDR approach is based on this flawed optimization setup it lacks technical rigor. In the following discussion, it is shown that URNDDR results are incorrect.

Consider state estimation of a scalar, linear, unconstrained, Gaussian process $x_{k+1}=A x_{k}+w_{k}, y_{k+1}=C x_{k+1}+v_{k+1}$, where $w_{k} \sim$ $N(0, Q)$ and $v_{k+1} \sim N(0, R)$. Given the estimate $\hat{x}_{k \mid k}$ and variance $P_{k \mid k}$. the predictions are: $\hat{x}_{k+1 \mid k}=A \hat{x}_{k \mid k}$ and $P_{k+1 \mid k}=A^{2} P_{k \mid k}+Q$. By solving the unconstrained optimization problem

$$
\min _{\hat{x}_{k+1 / k+1}} \frac{\left(y_{k+1}-C x_{k+1 \mid k+1}\right)^{2}}{R}+\frac{\left(x_{k+1 \mid k+1}-\hat{x}_{k+1 \mid k}\right)^{2}}{P_{k+1 \mid k}},
$$

Kalman filter (KF) corrector equations are obtained

$$
\begin{aligned}
\hat{x}_{k+1 \mid k+1} & =\frac{C P_{k+1 \mid k} y_{k+1}+R \hat{x}_{k+1 \mid k}}{C^{2} P_{k+1 \mid k}+R}, \\
P_{k+1 \mid k+1} & =\frac{P_{k+1 \mid k} R}{C^{2} P_{k+1 \mid k}+R} .
\end{aligned}
$$

The objective function may be justified using maximum likelihood, Bayesian inference, least squares or orthogonal projection among others. It can be shown that UKF reduces to KF for this case as it is expected. Define sigma points and weights at time $k$

$$
\begin{aligned}
& \hat{\chi}_{k \mid k, 0}=\hat{\chi}_{k \mid k} ; \quad W_{0}=\frac{\kappa}{1+\kappa}, \\
& \hat{\chi}_{k \mid k, 1,2}=\hat{\chi}_{k \mid k} \pm \sqrt{(1+\kappa) P_{k \mid k}} ; \quad W_{1,2}=\frac{1}{2(1+\kappa)} .
\end{aligned}
$$

The predictions are $\hat{\chi}_{k+1 \mid k, i}=A \hat{\chi}_{k \mid k, i}$, which lead to the predicted state and variance

$$
\begin{aligned}
& \hat{\chi}_{k+1 \mid k}=\sum_{i=0}^{2} W_{i} \hat{\chi}_{k+1 \mid k, i}=A \hat{x}_{k \mid k}, \\
& P_{k+1 \mid k}=\sum_{i=0}^{2} W_{i}\left(\hat{\chi}_{k+1 \mid k, i}-\hat{\chi}_{k+1 \mid k}\right)^{2}+Q=A^{2} P_{k \mid k}+Q .
\end{aligned}
$$

The sigma points are recalculated around the predicted state

$$
\begin{aligned}
& \hat{\chi}_{k+1 \mid k, 0}=\hat{\chi}_{k+1 \mid k}, \\
& \hat{\chi}_{k+1 \mid k, 1,2}=\hat{\chi}_{k+1 \mid k} \pm \sqrt{(1+\kappa) P_{k+1 \mid k}} .
\end{aligned}
$$

In their paper Vachhani et al. did not include this step, which makes Kalman gain incorrectly independent of $Q$. The original sigma points may be used if the state vector is augmented with noise terms. The measurement function is evaluated at sigma points as $\hat{\gamma}_{k+1 \mid k, i}=C \hat{\chi}_{k+1 \mid k, i}$ to compute the predicted measurement and the variance

$$
\begin{aligned}
& \hat{y}_{k+1}=\sum_{i=0}^{2} W_{i} \hat{\gamma}_{k+1 \mid k, i}=C \hat{x}_{k+1 \mid k}, \\
& P_{v v, k+1}=\sum_{i=0}^{2} W_{i}\left(\hat{\gamma}_{k+1 \mid k, i}-\hat{y}_{k+1}\right)^{2}+R=C^{2} P_{k+1 \mid k}+R .
\end{aligned}
$$

The covariance between predicted state and measurement is

$$
P_{x V, k+1}=\sum_{i=0}^{2} W_{i}\left(\hat{\chi}_{k+1 \mid k, i}-\hat{x}_{k+1}\right)\left(\hat{\gamma}_{k+1 \mid k, i}-\hat{y}_{k+1}\right)=C P_{k+1 \mid k} .
$$

The UKF filter gain is $K_{k+1}=P_{x v, k+1} / P_{v v, k+1}$. The state estimate and variance are

$\hat{x}_{k+1 \mid k+1}=\hat{x}_{k+1 \mid k}+K_{k+1}\left(y_{k+1}-\hat{y}_{k+1}\right)=\frac{C P_{k+1 \mid k} y_{k+1}+R \hat{x}_{k+1 \mid k}}{C^{2} P_{k+1 \mid k}+R}$, 
$P_{k+1 \mid k+1}=P_{k+1 \mid k}-K_{k+1} C P_{k+1 \mid k}=\frac{P_{k+1 \mid k} R}{C^{2} P_{k+1 \mid k}+R}$,

which is the Kalman filter.

Now consider the URNDDR approach for the same problem. After the sigma points are propagated we have $\hat{\chi}_{k+1 \mid k, 0}=\hat{x}_{k+1 \mid k}$ and $\hat{\chi}_{k+1 \mid k, 1,2}=\hat{\chi}_{k+1 \mid k} \pm A \sqrt{(1+\kappa) P_{k \mid k}}$. Note that the variance of these points is $A^{2} P_{k \mid k}$, which is not $P_{k+1 \mid k}$. The following least squares optimization problem is posed with respect to each sigma point although it is not apparent why they should be subject to it

$\min _{\hat{\chi}_{k+1 \mid k+1, i}} \frac{\left(y_{k+1}-C \chi_{k+1 \mid k+1, i}\right)^{2}}{R}+\frac{\left(\chi_{k+1 \mid k+1, i}-\hat{\chi}_{k+1 \mid k, i}\right)^{2}}{P_{k+1 \mid k}}$.

Since the objective function is of the same form, the solutions for "optimal" sigma points are in the form of Kalman filter corrector equation for the mean

$\hat{\chi}_{k+1 \mid k+1, i}=\frac{C P_{k+1 \mid k} y_{k+1}+R \hat{\chi}_{k+1 \mid k, i}}{C^{2} P_{k+1 \mid k}+R}$.

Hence, the "optimal" sigma points are rewritten as

$\hat{\chi}_{k+1 k+1,0}=\hat{x}_{k+1 k+1}$,

$\hat{\chi}_{k+1 \mid k+1,1,2}=\hat{\chi}_{k+1 \mid k+1} \pm \frac{R A \sqrt{(1+\kappa) P_{k \mid k}}}{C^{2} P_{k+1 \mid k}+R}$.
Computing the mean and covariance of the sigma points gives

$$
\begin{aligned}
\sum_{i=0}^{2} W_{i} \hat{\chi}_{k+1 \mid k+1, i}=\hat{x}_{k+1 \mid k+1}, & \\
\sum_{i=0}^{2} W_{i}\left(\hat{\chi}_{k+1 \mid k+1, i}-\hat{\chi}_{k+1 \mid k+1}\right)^{2} & =\frac{R^{2} A^{2} P_{k \mid k}}{\left(C^{2} P_{k+1 \mid k}+R\right)^{2}} \\
& =\left(P_{k+1 \mid k+1}-\frac{R Q}{C^{2} P_{k+1 \mid k}+R}\right)\left(\frac{R}{C^{2} P_{k+1 \mid k}+R}\right) .
\end{aligned}
$$

Although the mean matches the Kalman filter estimate, the variance is clearly something else. It appears like the variance obtained by URNDDR approach will be lower than the Kalman filter estimate. Hence, URNDDR does not reduce to the Kalman filter for this special case, which invalidates the optimization approach to compute the sigma points. In view of the growing awareness about UKF among process systems engineering community the authors are urged to address this issue. 